\title{
Recent developments of the SFXC software correlator
}

\section{A. Keimpema*}

Joint Institute for VLBI in Europe

P.O. Box 2, 7990 AA Dwingeloo, The Netherlands

E-mail: keimpema@jive.nl

\section{M.M. Kettenis}

Joint Institute for VLBI in Europe

P.O. Box 2, 7990 AA Dwingeloo, The Netherlands

E-mail: kettenisejive.nl

\section{S.V. Pogrebenko}

Joint Institute for VLBI in Europe

P.O. Box 2, 7990 AA Dwingeloo, The Netherlands

E-mail: pogrebenko@jive.nI

\section{A. Szomoru}

Joint Institute for VLBI in Europe

P.O. Box 2, 7990 AA Dwingeloo, The Netherlands

E-mail: szomoruejive.nI

The SFXC software correlator has become the primary correlator for the EVN. Due to its nature a software correlator is much more flexible than a hardware solution, greatly simplifying the development of new correlator features. SFXC offers a number of new features which were not previously available to EVN users. These new features include: Pulsar binning and gating, multiple simultaneous phase centres, arbitrary spectral resolution, and spectral windowing. Furthermore SFXC has a number of space science features such as Doppler tracking phase corrections and a near field delay model. Currently under development is a phased array mode which has applications for pulsar and fast transient search experiments. The resulting time series are stored in PSRFITS format, which is widely supported by pulsar analysis software.

11th European VLBI Network Symposium \& Users Meeting,

October 9-12, 2012

Bordeaux, France

${ }^{*}$ Speaker. 


\section{Introduction}

The origin of SFXC lies in the Cassini-Huygens mission to Titan. For this project a prototype software correlator was developed to track the descent of the Huygens probe through the atmosphere of Titan [1]. As part of the SCARIe and EXPReS projects the original Huygens code base was reimplemented as a distributed MPI application written in $\mathrm{C}++$. The correlator is intended to be deployed on a commodity computing cluster running a POSIX compliant operating system such as Linux. Currently most of the code base is written in C++ with many utilities written in Python.

SFXC is now the primary correlator for the EVN for both disk-based experiments and eVLBI. The correlator is deployed on a modern 384 CPU core Linux based cluster at JIVE. Data is played back using the Mark5 units. Thanks to the flexibility of a software correlator new features were added which were not available for EVN users before. In this paper we give an overview of the most important new features.

\section{Pulsar binning}

A crucial factor in pulsar observations is that for many pulsars the duty cycle, defined as the ratio between pulse width and pulse period, is relatively short. A significant improvement in signal to noise can be achieved by accumulating the correlation function only during pulse reception. This commonly is referred to as pulsar gating. The increase in signal to noise is approximately

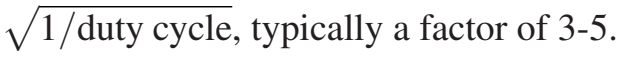

A closely related concept is pulsar binning. In pulsar binning the pulse period is divided into a number of time bins and the correlation function is accumulated individually for each bin. In SFXC the user specifies a binning interval and the bins are placed equidistantly within this interval. In this implementation pulsar gating is merely a special case of pulsar binning. The pulsar model is supplied by the user as a Tempo polyco file [2].

An important consideration in pulsar observations is the dispersive delay introduced by the interstellar medium. For some millisecond pulsars this dispersive delay can be a significant fraction of the pulsar period, even within a single sub-band. SFXC compensates for the dispersive delay using an incoherent de-dispersion algorithm [3].

In Fig. 1 we show an example pulse profile for B0329+54 using 100 time bins. The profile is obtained from the total power on the Effelsberg-Westerbork baseline.

\section{Multiple simultaneous phase centres}

Fundamentally, the field of view in a VLBI observation is limited only by the primary antenna beams of the participating stations. Provided that sufficient spectral and temporal resolution is available to keep smearing effects at an acceptable level it is possible to image the entire primary beam. However, traditional wide-field techniques run into practical issues as the resulting data 


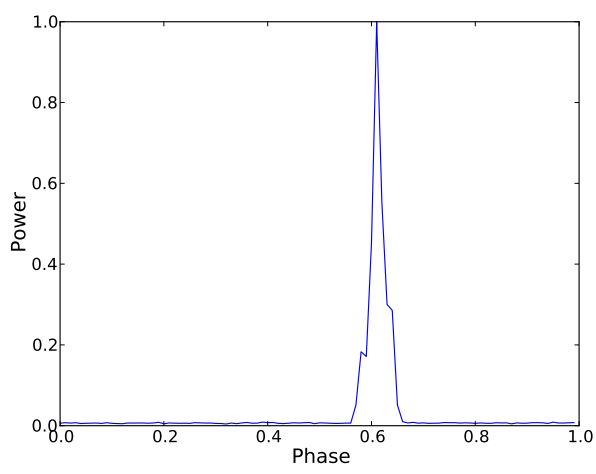

(a)

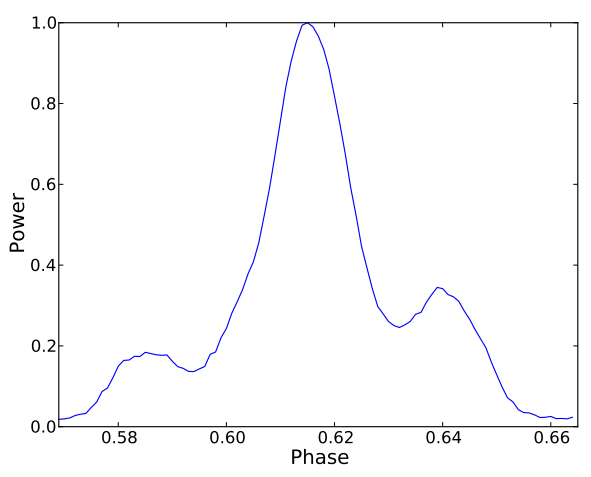

(b)

Figure 1: Pulse profile for B0329+54 obtained from 100 time bins. The profile is obtained from the total power on the Effelsberg-Westerbork baseline. The integration time was 600s. a) Whole period, b) Zoomed in on pulse

sets can become unwieldy large. E.g. to map the primary beam of a $100 \mathrm{~m}$ station at $21 \mathrm{~cm}(9.5$ arcmin) in a typical 10 station EVN experiment would require require 100ms integrations and 7.8 khz channel widths to keep smearing losses within $10 \%$. This results in a dataset of approximately 3 TB over the course of 6 hours. However, on average there will be only a few dozen mJy class sources present within the beam of a $100 \mathrm{~m}$ dish. Therefore a more practical approach is to produce a separate narrow field data set for each source in the field rather than a single monolithic wide field data set. This greatly simplifies the data reduction. This method is known as correlating with multiple simultaneous phase centres.

Internally the correlator correlates the data at the required spectral and temporal resolution. After each sub integration the phase center is then shifted to each point of interest. The visibilities for each point of interest are then accumulated individually and averaged down in time and frequency before they are written to disk [4].

Because the phase shifts are applied relatively infrequently the performance scales very well with the number of phase centres. A typical example would be imaging the entire primary beam of a 100 meter dish. A benchmark for this particular example using 10 stations shows an increase in correlation time by nearly $50 \%$ when multiple phase centres is enabled. The correlator parameters were chosen to keep smearing losses within $1 \%$. The performance loss is mostly due to the large internal spectral resolution. Each additional phase center, however, comes at very little cost. Going from 2 to 100 phase centres increases correlation time by a mere $25 \%$ in this case.

\section{Spacecraft tracking}

A typical phase referencing spacecraft tracking experiment alternates the antenna pointing between the narrow band signal of the spacecraft and a broad band signal of an astronomical calibrator 


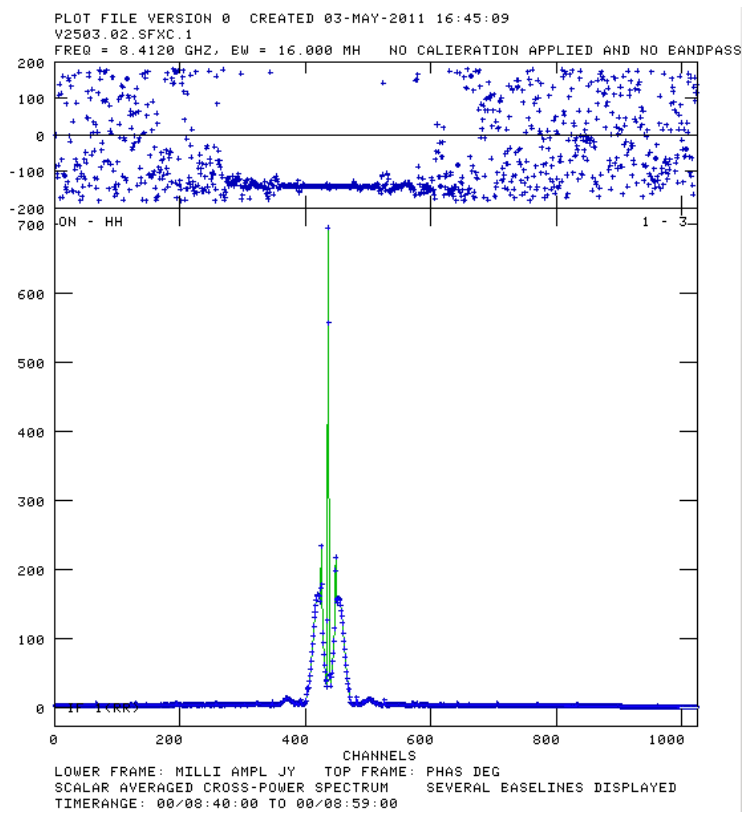

Figure 2: Spectrum of the ESA Venus EXpress (VEX) spacecraft signal at $8.4 \mathrm{Ghz}$. The distance to the target was $1.3 \mathrm{AU}$.

source. The phase solutions of the correlation results for the calibrator are then transferred to the correlation results of the spacecraft signal. Because the calibrator is located in the far-field, and the spacecraft in the near-field, two delay models are needed. We use the far-field and near-field models by Duev et al. [5]. Both delay models use the same station positions, Earth rotation and tidal deformation, atmospheric, and general relativity correction models.

Residual to the delay compensation there is a frequency drift in the carrier signal caused by the Doppler shift due to the spacecraft motion as well as an intrinsic drift in the electronics of the spacecraft. The correlation becomes a two-pass process where first the frequency drift is determined which is then used as an input for the correlator in the second pass.

In Fig. 2 we show a spectrum of the ESA Venus EXpress (VEX) spacecraft signal at 8.4 Ghz. The distance to the target was $1.3 \mathrm{AU}$. The near field delay effect before correction was approximately $10 \mu \mathrm{s}$. This was reduced to a $\sim 1$ ns stochastic delay accuracy at correlation.

\section{Phased array}

The most recent feature added to SFXC is a phased array mode. This mode can be used in pulsar and fast transient searches. In phase array mode the signal voltages are coherently summed rather than correlated. The coherent addition requires accurate calibration information to be available for all participating stations. The calibration information is obtained by first correlating the whole experiment like a conventional VLBI observation. The usual calibration steps are then applied 


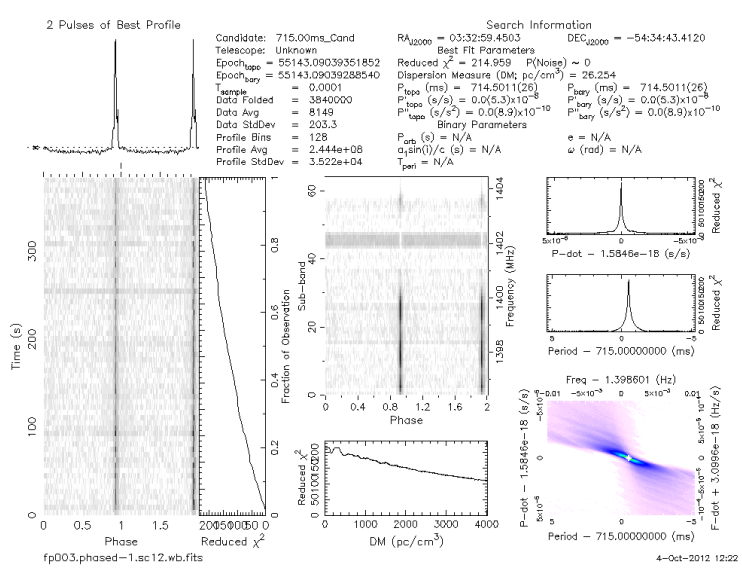

Figure 3: Profile of pulsar B0329+54 obtained by folding a time series produced using the phased array mode of SFXC. The image was created using the pulsar search suite PRESTO.

using the data reduction package AIPS ${ }^{1}$. The resulting calibration tables are then exported back to the correlator and are applied during the addition of signal voltages.

Pulsar searches are performed by finding periodicities in the signal power [6]. Similarly short transient searches locate short increases in signal power. Working with power spectra rather than voltages has the advantage that signal powers can be integrated greatly reducing the data volume. For pulsar searches integration times are typically $10-100 \mu \mathrm{s}$. The floating point power spectra are re-quantized to 8 bits and stored in PSRFITS format. The PSRFITS data format is widely accepted by pulsar processing software such e.g. PRESTO [7].

In Fig. 3 we show a profile of pulsar B0329+54 obtained using the pulsar search suite PRESTO. For this image the data streams of stations Westerbork, Effelsberg, Torun, Onsala, and Jodrell Bank were coherently summed using the phased array mode.

\section{Conclusion}

SFXC has become the production correlator of the EVN. The flexibility of a software correlator makes it much easier to implement new correlator features compared to a hardware solution. Many new features were implemented in SFXC which were not previously available for the EVN before such as: pulsar binning and gating, multiple simultaneous phase centres, and near field space science. The most recent feature, the phased array mode, will open new applications in pulsar and fast transient searches with the EVN.

\section{Disclaimer}

Research leading to these results has received funding from the European Union's Seventh Framework Programme (FP7/2007-2013) under grant agreement $n^{\circ}$ RI-261525 NEXPReS. This material

\footnotetext{
${ }^{1}$ http: //www.aips.nrao.edu
} 
reflects only the author's views, and the European Union is not liable for any use that may be made of the information contained therein.

\section{References}

[1] S.V. Pogrebenko, et al., VLBI tracking of the Huygens probe in the atmosphere of Titan, Planetary Probe Atmospheric Entry and Descent Trajectory Analysis and Science, 544, 197 (2004).

[2] G.B. Hobbs, R.T. Edwards, and R.N. Manchester, TEMPO2, a new pulsar-timing package, MNRAS, 369, 655 (2006).

[3] F.A. Jenet, W.R. Cook, T.A. Prince, and S.C. Unwin, A Wide-Bandwidth Digital Recording System for Radio Pulsar Astronomy, PASP, 109, 707 (1997).

[4] A.T. Deller, et al., DiFX-2: A More Flexible, Efficient, Robust, and Powerful Software Correlator, PASP, 123, 275 (2011).

[5] D.A. Duev, G. Molera Calvés, S.V. Pogrebenko, L.I. Gurvits, G. Cimó, and T. Bocanegra Bahamon, Spacecraft VLBI and Doppler tracking: algorithms and implementation, A\&A, 541, A43 (2012).

[6] D. Lorimer and M. Kramer. Handbook of Pulsar Astronomy, Cambridge university press, 2005.

[7] S.M. Ransom, New search techniques for binary pulsars, Ph.D. theses, Harvard University, 2001. 